\title{
Design of coherence-aware channel indication and prediction for rate adaptation
}

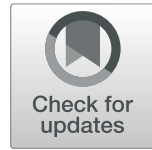

Yongjiu Du ${ }^{1}$, Pengda Huang ${ }^{2}$, Yan Shi ${ }^{*}$ (D) Dinesh Rajan ${ }^{3}$ and Joseph Camp ${ }^{3}$

\begin{abstract}
A number of rate adaptation protocols have been proposed using instantaneous channel quality to select the physical layer data rate. However, the indication of channel quality varies widely across platforms from simply a received signal strength level to a measurement of signal-to-noise ratio (SNR) across sub-carriers, with each channel quality indicator having differing levels of measurement error. Moreover, due to fast channel variations, even aggressive channel probing fails to offer an up-to-date notion of channel quality. In this paper, we propose a coherence-aware Channel Indication and Prediction algorithm for Rate Adaptation (CIPRA) and evaluate it analytically and experimentally, considering both the effects of measurement errors and the staleness of channel quality indicators. CIPRA uses the minimum mean square error (MMSE) method and first-order prediction. Our evaluation shows that CIPRA jointly considers the time interval over which the prediction will occur and the coherence time of the channel to determine the optimal window size for previous channel quality indicator measurements. Also, we demonstrate that CIPRA outperforms existing methods in terms of prediction fidelity and throughput via experimental results. By combining a strong channel indicator with the coherence-aware MMSE first-order channel prediction algorithm, CIPRA nearly doubles the throughput achieved in the field from the indication and prediction method currently used by off-the-shelf WiFi interfaces.
\end{abstract}

Keywords: Rate adaptation, Prediction, Channel indication, Channel coherence, MMSE

\section{Introduction}

Channel fluctuations often exist in wireless communication systems and present great challenges in selecting the best data rate or modulation coding scheme (MCS) for communication. When there is a change in direction of the transmitter/receiver, antenna elevation and polarization, interference from nearby devices, or scatter distributions, the channel quality can vary, resulting in fluctuations in signal reception, even within the same environment. Depending on the magnitude of the variation, the received signal strength (RSS) can drastically change the link performance [1-4]. Rate adaptation protocols can be implemented to combat the fading channels and achieve high spectrum efficiency by dynamically changing the data rate according to the channel quality.

Rate adaptation protocols that depend upon packet success/failure information have been implemented in

*Correspondence: shiy@smu.edu

${ }^{3}$ Department of Electrical Engineering, Southern Methodist University, Dallas, TX, USA

Full list of author information is available at the end of the article commercial equipment and widely discussed [5-10]. However, these loss-based protocols usually require tens of data frames to develop a reasonable estimate of the channel conditions. Other factors such as hidden terminals and interference can lead to inappropriate conclusions about the causes for the losses [11]. Thus, for fast-fading channels (e.g., in vehicular networks), these protocols cannot accurately track the changing channels, resulting in wrong selections of MCS. Moreover, packetlevel estimation is too coarse to achieve an accurate estimation of the instantaneous channel conditions. With interfering sources, the transmitter can not distinguish the reason for packet loss and jitter (i.e., whether it is from a poor channel or interference). These factors contribute to frequent under-selection of the transmission rate by loss-based protocols [11].

To enable fast-fading channel tracking, various channelindicator-based rate adaptation protocols have been proposed [12-15]. It is well known that for a certain MCS, there is a theoretical bit error rate (BER) versus signalto-noise ratio (SNR) relationship [16]. SNR values can be 
reported to the transmitter at the physical layer (PHY)frame level to enable selection of the optimal rate in fastfading channels. Some of the SNR-based rate adaptation protocols leverage the received signal strength indicator (RSSI) to calculate the SNR. However, for different commercial wireless network devices, the RSSI varies significantly for the same received signal strength. Moreover, in the presence of interference and noise, the estimates of the signal power reported from RSSI measurements can be highly distorted [17]. In [13], the authors propose a SoftRate metric to indicate channel quality and select the optimal rate for transmission. However, this scheme requires a soft-in, soft-out decoder to calculate the SoftRate indicator, which is not available for most of the existing network transceivers and may also be resourceintensive for the design of future transceivers. AccuRate, a constellation-quality based indicator is provided in [15]. Nevertheless, the hardware cost and complexity of AccuRate is also high, often precluding the calculation of the channel indicator in the implementation. The Effective SNR metric [14] leverages the channel response in the frequency domain and the noise variance on each sub-carrier to predict the best rate to transmit a packet. The information required is already available in several wireless network interfaces.

In our previous work [12], we consider RSSI as the unique channel indicator under limited comparison matrices, which has been shown to be inaccurate and inconsistent across vendors [13]. In this work, in addition to the original channel indicator of RSSI, we study and analyze two more advanced channel indicators: SNR and Effective SNR. We implement channel prediction and rate adaptation based on these two advanced metrics to evaluate their performance in combination with traditional channel prediction methods and our proposed channel prediction method, showing significant throughput improvement. We additionally analyze the prediction error performance for our proposed algorithm at various time intervals, predicting the importance of our work on next-generation protocol design with higher frequency bands and tighter timing parameters. At last, we implement the Minstrel rate adaptation and compare our proposed rate adaptation method with it, showing that our proposed rate adaptation method outperforms Minstrel significantly.

Typically, in SNR-based rate adaptation, the receiver decides the rate of the next transmitted packet according to the measured SNR of the current packet, whether the measurement originates from the RTS/CTS or DATA/ACK exchange [11]. Several potential problems exist with this mechanism. First, if the channel changes quickly or there is a large time interval between two adjacent packets, the SNR reported by the last transmission may not accurately represent the instantaneous channel quality. Second, the SNR reported during the last transmission may not be accurate due to measurement errors. Even for slowly varying channels, there might be rate over-selection or under-selection due to channel quality estimation errors.

The rate selection problems caused by channel quality estimation errors have been studied [16, 18]. In order to increase the accuracy of rate selection, both works as leverage-filtering techniques to reduce channel quality estimation errors. However, the delay incurred in filtering can make rate selection even more stale to track with ongoing channel quality changes. To address the channel quality staleness problem, channel prediction has been extensively studied [19-23]. However, most work assumes perfectly accurate channel measurements to predict the future channel state, which may not be possible in hardware. Moreover, the estimation error can make the prediction highly erroneous if using the reported value from the last transmission.

In this paper, we propose a coherence-aware MMSE first-order prediction algorithm that takes into account both the measurement inaccuracy and measurement staleness. Prediction intervals and channel coherence time are jointly considered to select the optimal size of prediction window. We perform simulation studies as well as in-field experimentation on emulated and in-field channels, respectively. For our analysis, we implement an IEEE 802.11 PHY system on WARP (Wireless Open-Access Research Platform), an field programmable gate array (FPGA)-based platform [11]. We also implement and compare three different channel indicators, RSSI, SNR, and Effective SNR, and combine each with the proposed prediction algorithm to investigate the indicator's impact on the performance of channel prediction. To generate repeatable channel effects, we test our design on a channel emulator and compare the three indicators in diverse channel conditions. In addition to lab experiments, we also conduct field tests to show the in situ throughput improvements provided by our algorithm.

The main contributions of our work are as follows:

1 We propose a coherence-aware MMSE first-order channel quality prediction algorithm, which takes into account both the measurement errors and staleness of the channel quality. This scheme adapts the measurement processing parameters to the Doppler shift to achieve good performance under various mobility scenarios.

2 We implement and evaluate a family of the most commonly used channel quality indicators for rate adaptation, including RSSI, SNR, and Effective SNR.

3 We analyze different channel prediction approaches and compare them in terms of prediction errors as well as over-selection and under-selection probabilities for rate adaptation. 
4. We present and implement a Doppler shift estimation method based on LCR (level-crossing rate) with a homogeneous window to remove the effect of channel quality measurement errors, achieving a good balance of complexity and accuracy.

5 We implement the existing channel prediction algorithm and the proposed algorithm on WARP and experimentally compare them in terms of system throughput through both repeatable channel emulator tests and in-field experiments. By combining Effective SNR with the coherence-aware MMSE first-order channel prediction algorithm, channel indication and prediction for rate adaptation (CIPRA) achieves up to a 98\% throughput improvement in the field over the indication and prediction method currently used in off-the-shelf cards.

The rest of this paper is organized as follows. Section 2 introduces the online Doppler shift estimation method and discusses various channel quality indicators. Then, we describe the framework of our proposed CIPRA algorithm and compare its performance with conventional prediction algorithms in Section 3. In Section 4, we introduce the hardware setup for CIPRA evaluation and provide numerical results in Section 5. Finally, we conclude our work in Section 6.

\section{Background and related work for channel indication and prediction}

In this section, we first introduce the channel fading model used in this work. Then, we describe a Doppler shift estimation method for our platform implementation.
Finally, in addition to the typical performance metrics developed to indicate the channel quality, such as RSSI and SNR, we study and analyze a more advanced channel quality indicator: Effective SNR.

\subsection{Channel characteristics}

Wireless channel quality is often affected by changing environments and interference. With the transmit signal power fixed, channel quality can be quantified by the received signal quality. We use a Rayleigh fading channel model in the following analysis and simulation. The normalized auto-correlation function, $R(\tau)$, of a Rayleigh fading channel with motion at a constant velocity is a zeroth-order Bessel function of the first kind [24, 25]:

$$
R(\tau)=J_{0}\left(2 \pi f_{d} \tau\right)
$$

Here, $\tau$ is the time delay, and $f_{d}$ is the maximum Doppler shift. The auto-correlation functions of a Rayleigh fading channel with a maximum Doppler shift of $10 \mathrm{~Hz}$ and $20 \mathrm{~Hz}$ are shown in Fig. 1. This auto-correlation reflects the statistical dependence between the channel gains at different times, which is leveraged in the prediction.

\subsection{Online doppler shift estimation}

In this work, we introduce a Doppler shift estimation method that we implement and evaluate on our hardware platform. This method can be applied to our channel prediction algorithm discussed in Section 3. In general, Doppler shift estimation can leverage channel estimates, LCR, a maximum likelihood function, or correlation function [26]. LCR-based Doppler shift estimation achieves

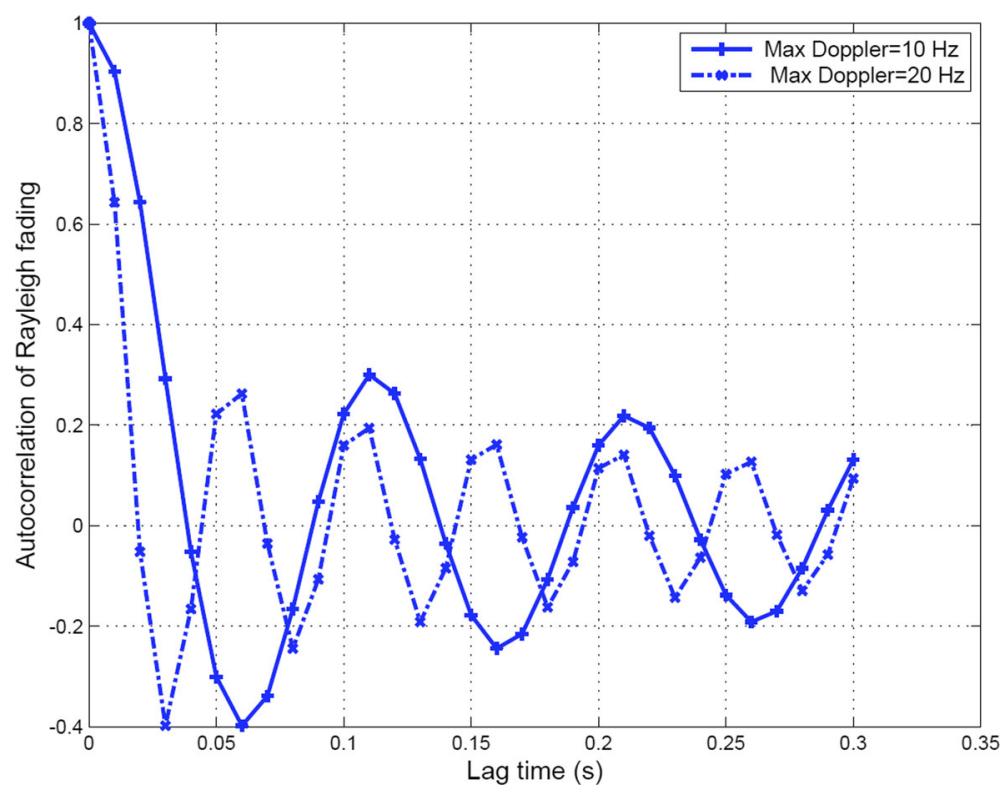

Fig. 1 Auto-correlation function of a Rayleigh fading channel with a maximum Doppler shift of $10 \mathrm{~Hz}$ 
a good balance between complexity and accuracy. For Rayleigh fading channels, LCR is expressed as [27]:

$$
\mathrm{LCR}=\sqrt{2 \pi} f_{d} \rho e^{-\rho^{2}}
$$

Here, $\rho$ is the threshold normalized to the root mean square (RMS) signal level [27]. For a fixed Doppler shift, the LCR achieves its maximum value, $\mathrm{LCR}_{\max }$, when $\rho=$ $\sqrt{0.5}$, and is given by

$$
\mathrm{LCR}_{\max }=\sqrt{\pi} e^{-0.5} f_{d}
$$

In hardware, the received signal is also corrupted by additive noise. The LCR resulting from the noise usually leads to over-estimation of the channel level-crossing rate. In [28], an fast Fourier transform (FFT)-based Doppler-adaptive noise suppression method is proposed to remove the effect of additive noise on LCR-based Doppler shift estimation. However, the FFT/IFFT processing is computationally expensive, requiring approximately $\frac{34}{9} N \log _{2} N$ real multiplications and additions for an $N$-point FFT/IFFT [29]. In this paper, we create a homogeneous-window method to avoid over-estimation caused by the additive noise. The main steps to this method are the following:

1 Pick a threshold value from a pre-defined threshold set and compare the RSSI samples with this threshold. If the RSSI value of sample $i$ is greater than the selected threshold for each indicator, $c_{i}=1$.
Otherwise, $c_{i}=0$, meaning that the RSSI is below the selected threshold.

2 Apply a sliding time window $\tau$ to the results in step 1 . If $c_{i}==1$ for all the samples in window $\tau$, we denote the system state $S_{i}=1$. If $c_{i}==0$ for all the samples in window $\tau$, we denote the system state $S_{i}=-1$. Otherwise, $S_{i}=0$. For multiple adjacent system state samples with the same value, only record one sample.

3 Calculate the derivative of the state vector recorded in step 2 and count the number of transitions of the derivative from negative to positive in one second, denoted by $n$.

4. Repeat step 1 to step 3 for all the values in the pre-defined threshold set, and finally, find the maximum value of $n$.

To decide the time window $\tau$, we jointly consider the RSSI sample period and the Doppler shift range we want to estimate. In the IEEE 802.11 standard, the RSSI is reported every packet. For the maximum Doppler shift range of 10 to $100 \mathrm{~Hz}, \tau$ of $3 \mathrm{~ms}$ achieves a normalized square error of 0.003 in our experiments using the channel emulator. Figure 2 shows the Doppler shift estimation by using the WARP board and the channel emulator (both are described in Section 5).

\subsection{Channel quality indicators}

\subsubsection{RSSl: a poor channel indicator}

Wireless channel quality is often affected by changing environments and interference. With the transmit signal power fixed, channel quality can be evaluated by the

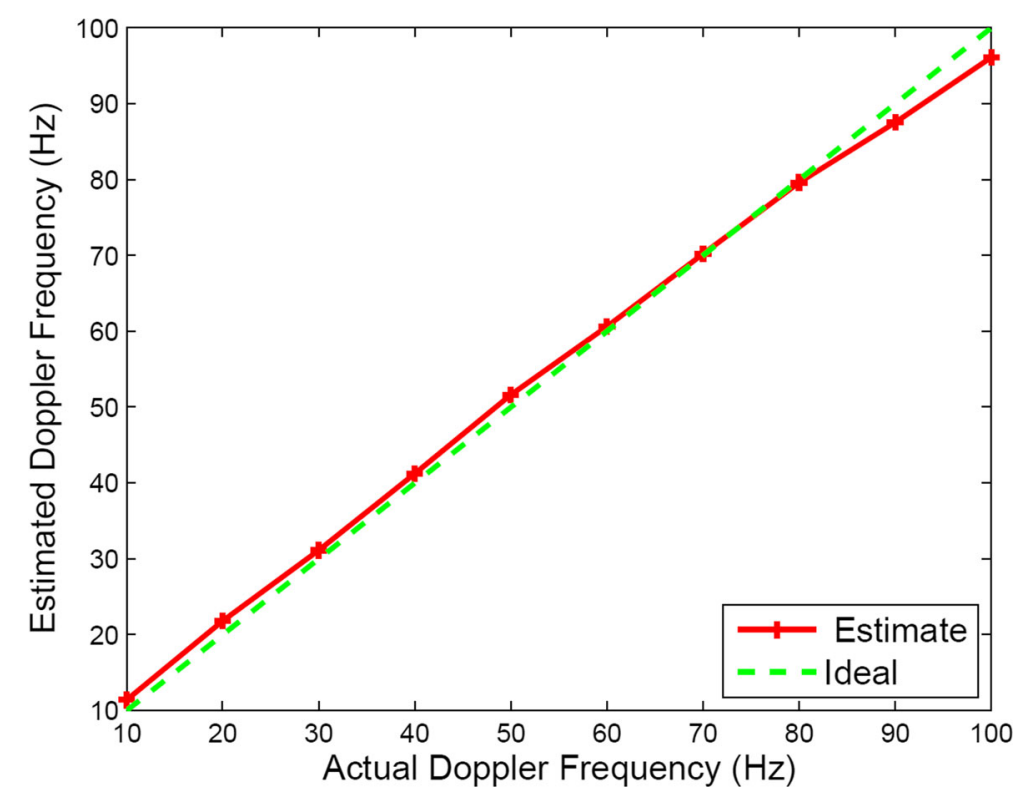

Fig. 2 Doppler shift estimation on WARP 
received signal quality. The most accessible channel quality indicator is RSSI [17]. RSSI is a relative value with off-the-shelf devices in which vendors usually use arbitrary scales from 0 to maximum-RSSI, where maximumRSSI is vendor-specific. RSSI is often not associated with any particular power scale and not required to be of any particular accuracy or precision [30]. Hence, the received signal strength numbers reported by a network interface are inconsistent across vendors and should not be assumed to be representative of a particular channel state.

We now carry out experiments to evaluate the reliability of using RSSI as the channel indicator. We use one WARP as the transmitter to send a signal with $10 \mathrm{MHz}$ bandwidth and $-62 \mathrm{dBm}$ power and directly connect it to another WARP receiver with a coaxial cable. The reported RSSI values from our WARP receiver are shown in Fig. 3. We can see that, even with the same transmit power and channel state, the reported RSSI values can vary as much as $14 \mathrm{~dB}$. Even if we use a filter to reduce the variance of the reported RSSI, other factors may also greatly affect the RSSI-based channel quality prediction accuracy. The general system architecture for the signal path is shown in Fig. 4. Any error or interference generated by the components in group 1 may vastly affect the received BER, but may not affect the RSSI value. Any error or interference generated by the components in group 2 may affect the RSSI value, but may not significantly affect the BER. Therefore, for different transmitter and receiver pairs, system components internally have different performances. The factors that might affect the channel quality estimation accuracy using RSSI include the following:
1 Phase noise. With the same RSSI, there might be different phase noises caused by the jitter of the clocks on both the transmitter and the receiver, leading to different values of BER. However, the phase noise may not affect the received signal power level.

2 Amplifier non-linearity. For orthogonal frequency-division (OFDM) systems, the non-linearity of both the transmit amplifier and the receive amplifier often cause inter-carrier interference (ICI) [31]. ICI may not affect the RSSI, but often affects the received BER performance.

3 RSSI signal noise. RSSI is measured in the transceiver and output in the form of an analog signal, which often suffers from noise and interference on the board.

4 RSSI analog to digital converter (ADC) performance. In a system, an ADC is typically used to convert the RSSI signal from the analog to digital domain. The noise on the board, the resolution of the ADC, and the reference voltage stability of the ADC may all affect the digitized RSSI value.

5 RSSI sample duration. In the IEEE 802.11 standard, RSSI is calculated during the preamble of a PHY frame. The limited duration of the preamble can not guarantee an accurate RSSI estimate.

Considering all the effects listed above, with the same received BER, the receivers often report different RSSI values for different transmitter and receiver pairs. We compare the RSSI values reported (shown in Fig. 5) from two different receivers but with the same signal source

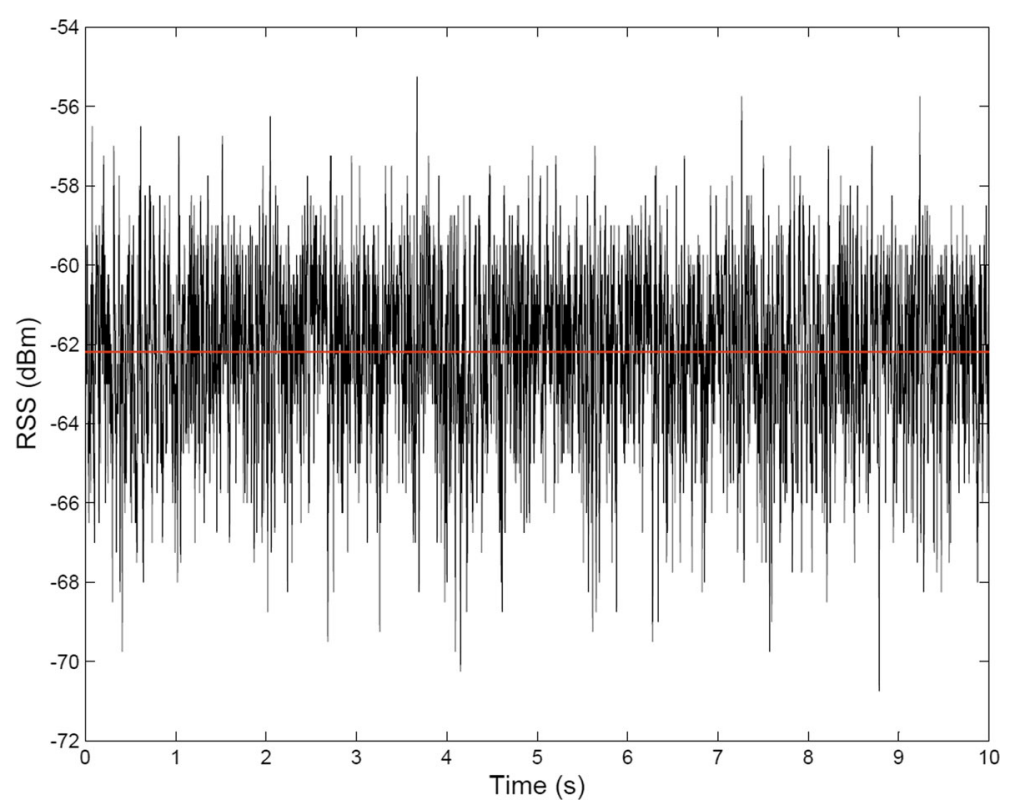

Fig. 3 Raw RSS values reported with the same transmit power and channel gain 


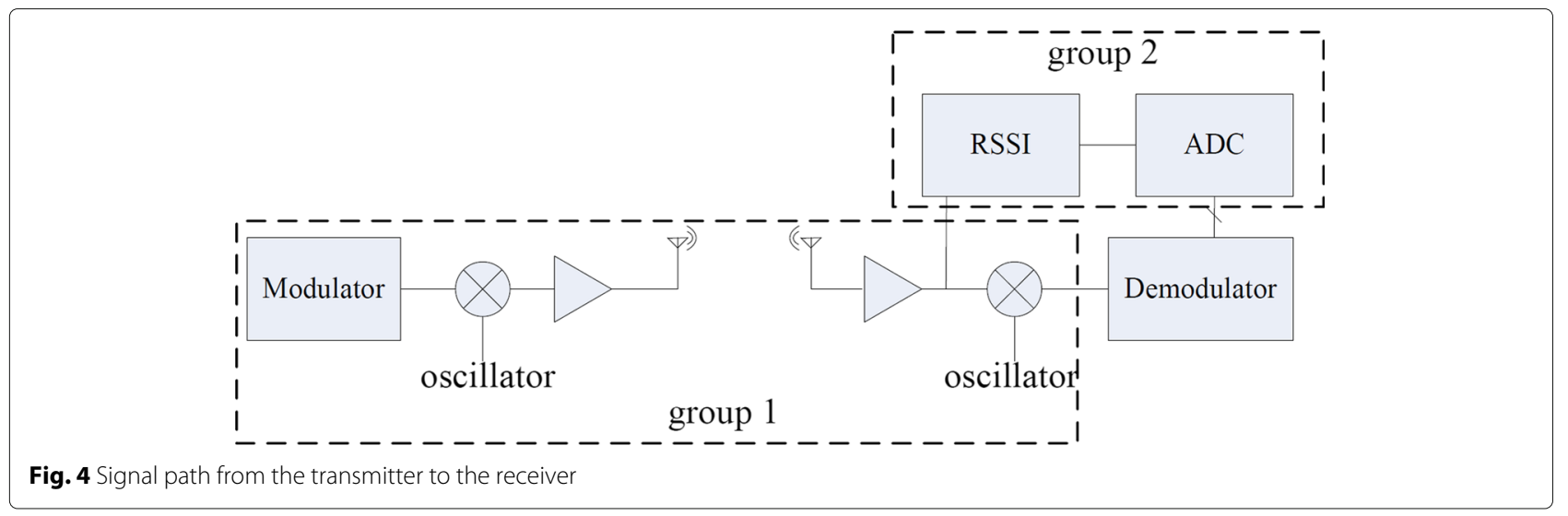

and emulated channel. Even with the same received signal, there is about 1.5-dB difference of reported RSSI between receivers on average. As a result, the RSSI inconsistency may severely handicap an optimal rate selection decision.

\subsubsection{SNR: a more reliable channel indicator}

Most of the soft decoders need the SNR or noise variance to calculate the decision probabilities of the demodulated signals. There are diverse SNR estimation methods for OFDM systems. In [32], the author proposes iterative SNR estimation based on pilot sub-carriers in an 802.11 n system. In [33], an algorithm based on finding the difference between a noisy received sample in the frequency domain and the best hypothesis of the noiseless sample is proposed to estimate the SNR. In this work, we implement an SNR estimation method based on the Schmidl-Cox algorithm [34] because of its high accuracy and low complexity. In addition, we use the IEEE 802.11 PHY frame as the frame structure in this work, as shown in Fig. 6 . One frame is composed of a preamble, a header symbol, and the number of OFDM symbols forming the data payload. One preamble consists of one short preamble and one long preamble [35]. In our design, we take advantage of the known training symbols (two identical OFDM symbols with a $1 / 2$ symbol prefix) in the long preamble to calculate the SNR of the received packet. A detailed calculation can be found in the Appendix 1. We show the SNR distribution plotted in Fig. 7. The SNR has a mean value of $16 \mathrm{~dB}$, with a standard deviation of $0.91 \mathrm{~dB}$. Comparing the RSSI distribution which has a standard deviation of $1.5 \mathrm{~dB}$, we can show that SNR is more accurate than the RSSI as the channel quality indicator.

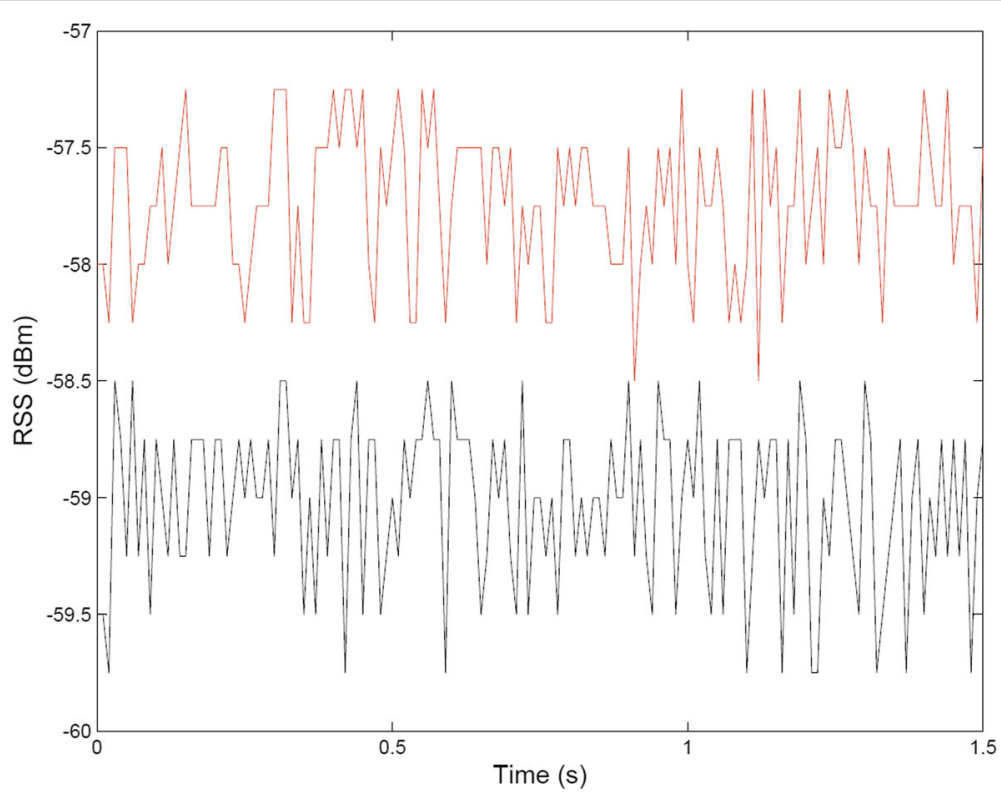

Fig. 5 RSS values reported by different receivers (one shown above the other) with the same level of achieved BER 


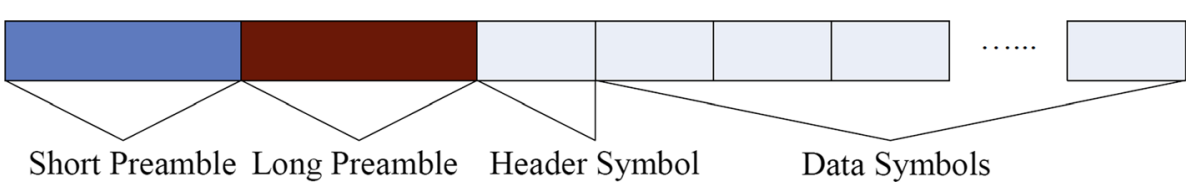

Fig. 6 IEEE 802.11 PHY frame structure

\subsubsection{Effective SNR: a robust channel indicator}

Although the SNR estimation is more tightly bounded and consistent with the channel state than the RSSI, a frequency-selective fading channel may disturb the mapping from SNR to BER. However, in a multi-path channel, the frequency-selective characteristics may result in a higher BER than a flat-fading channel with the same SNR, which corresponds to a lower Effective SNR than the actual SNR (demonstrated in Fig. 8). To solve this problem, Halperin [14] proposed the Effective SNR metric to improve the rate selection accuracy in multi-path fading channels. Instead of averaging the SNR on all the sub-carriers for SNR-based rate adaptation algorithms, systems implementing the Effective SNR not only average the BER on all the sub-carriers but also obtain an equivalent SNR with the same BER as narrow-band systems. This process can be represented by [14, Eqs. (1) and (2)]:

$$
\begin{gathered}
\mathrm{BER}_{e f f, k}=\frac{1}{52} \sum_{s=1}^{S} \operatorname{BER}_{k}\left(\rho_{s}\right) \\
\rho_{e f f, k}=\mathrm{BER}_{k}^{-1}\left(\operatorname{BER}_{e f f, k}\right)
\end{gathered}
$$

Here, $\rho_{s}$ is the SNR on sub-carrier $s$. Assuming the average SNR is $\rho$ and the channel gain on sub-carrier $s$ is $H_{s}$, then $\rho_{s}$ can be calculated as $\rho_{s}=\rho \frac{\left|H_{s}\right|^{2}}{|\bar{H}|^{2}}$, where $|\bar{H}|^{2}$ is the mean square of the channel gain across all the sub-carriers.

In an additive white Gaussian noise (AWGN) channel, the relationship between SNR and BER varies among different modulations [14, 36], as shown in Table 1. In the following discussion and experimental evaluation, we see that Effective SNR outperforms the other channel indicators due to its ability to capture both time-selective and frequency-selective fading effects.

\section{Design of CIPRA}

In this section, we analyze different channel prediction algorithms and propose an advanced algorithm to keep the transmitter in step with the fluctuating channel quality. In order to improve prediction accuracy, we take into account both the measurement error of the channel indicator and the staleness of the channel quality reported by the receiver.

\subsection{Existing prediction methods}

The wireless channel usually changes continuously and randomly with time, which makes the implementation of accurate closed-form characterization challenging. Nevertheless, a Rayleigh fading channel model is a good

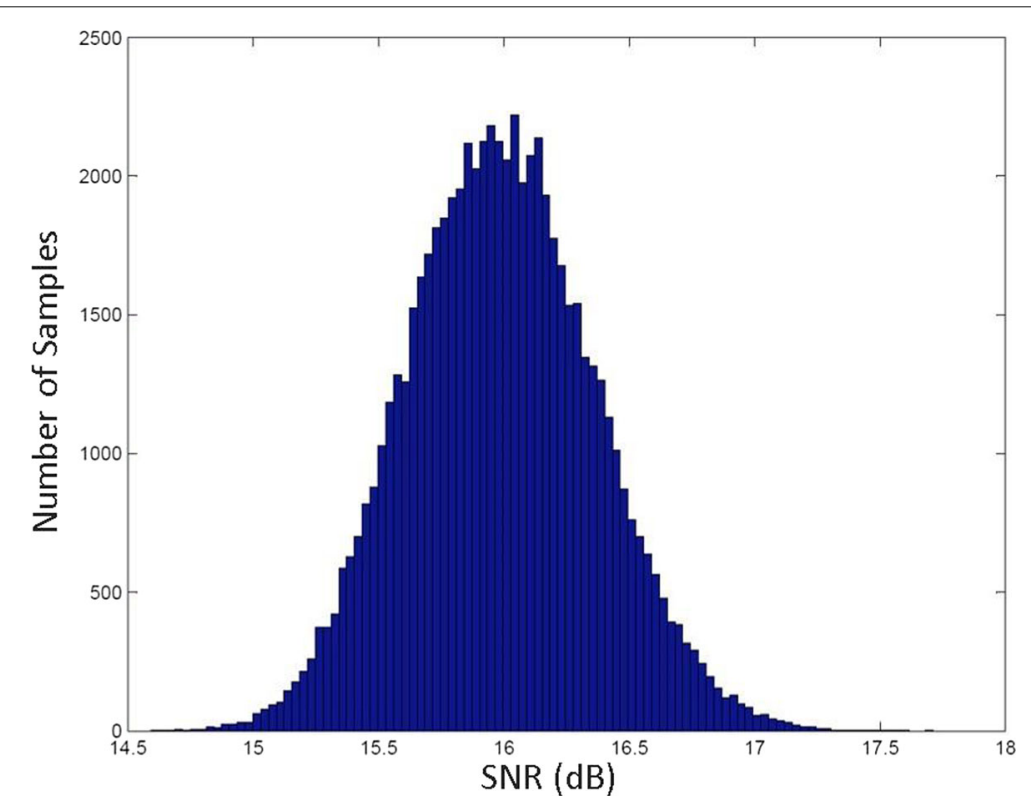

Fig. 7 Distribution of estimated SNR 


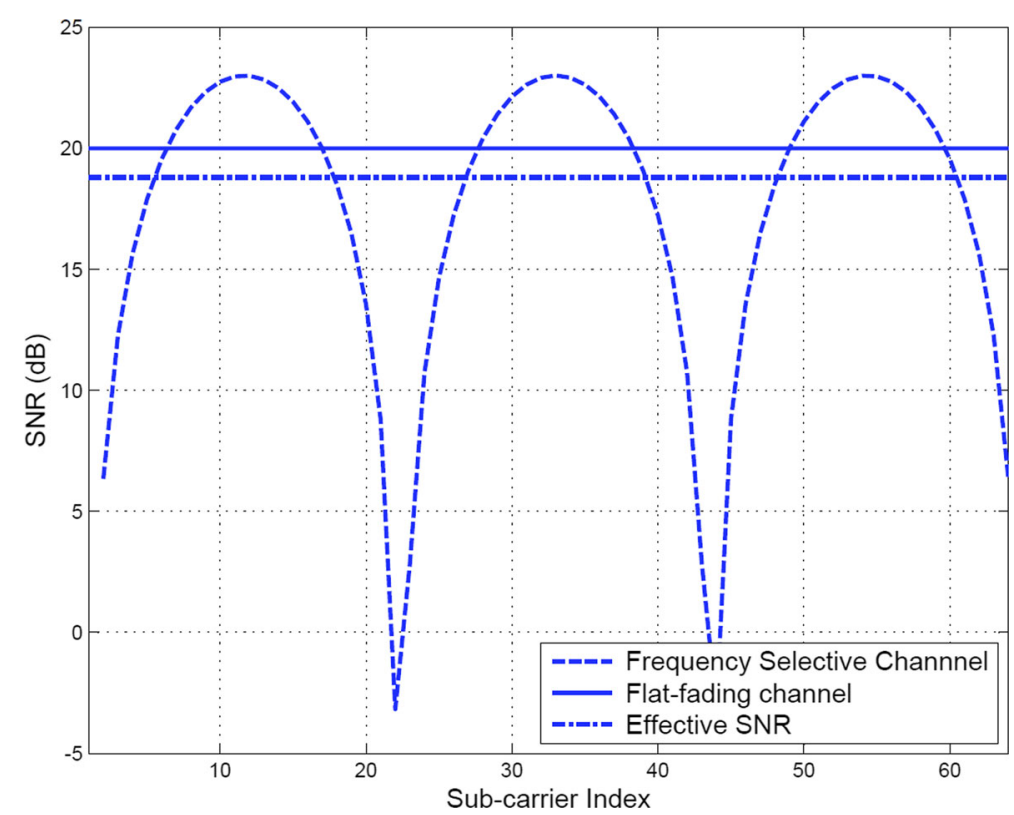

Fig. 8 Different frequency-selective patterns with the same SNR value

approximation and agrees well with empirical observations for mobile communications [25]. In order to select the optimal rate, the transmitter constantly needs the channel quality measurement from the receiver. Prior SNR-based protocols have frequently used the channel quality measured from the last packet transmission to the pertinent receiver [18]. Whether the last packet is a probe packet from the RTS/CTS exchange or simply the last data packet, these channel indicator measurements are stale in fast-fading channels. There are several mechanisms to make full use of the previous channel quality measurements for rate adaptation.

\subsubsection{Follower}

For this mechanism, the transmitter simply copies the channel measurements from the last packet transmission as the predicted value of the ongoing channel quality [18]. In particular, Follower can simply be denoted as

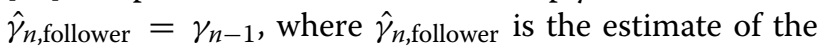
ongoing channel quality and $\gamma_{n-1}$ is the channel quality measurement reported during the last packet transmission. This estimate has minimal complexity but suffers

Table 1 BER function with different modulations

\begin{tabular}{ll}
\hline Modulation & $B E R_{k}(\rho)$ \\
\hline BPSK & $Q(\sqrt{2 \rho})$ \\
QPSK & $Q(\sqrt{\rho})$ \\
16-QAM & $\frac{3}{4} Q\left(\sqrt{\frac{\rho}{5}}\right)$ \\
64-QAM & $\frac{7}{12} Q\left(\sqrt{\frac{\rho}{21}}\right)$ \\
\hline
\end{tabular}

from both measurement errors and staleness of the past channel indicator values.

\subsubsection{Moving Average}

There are three main kinds of moving average methods: simple moving average, linear weighted moving average (LWMA), and exponential weighted moving average (EWMA) [23]. Simple moving average is the unweighted mean of the previous points in a window size of $w$. The estimated channel quality $\hat{\gamma}_{n, \mathrm{MA}}$, is denoted as

$$
\hat{\gamma}_{n, \mathrm{MA}}=\frac{\gamma_{n-1}+\gamma_{n-2}+\cdots+\gamma_{n-w}}{w}
$$

Simple moving average method reduces the effect of the measurement errors, while making the staleness effect more serious than the Follower method.

For LWMA, weight factors are assigned to the past measurements in a linear progression with a window size of $w$. The estimated value $\hat{\gamma}_{n, \mathrm{LWMA}}$, can be expressed as

$$
\hat{\gamma}_{n, \text { LWMA }}=\frac{w \gamma_{n-1}+(w-1) \gamma_{n-2}+\cdots+\gamma_{n-w}}{(w+1) w / 2}
$$

LWMA puts greater weight on more recent measurements, which results in a balance between the prediction staleness and the measurement errors.

For EWMA, the weight of the measurements decreases by a factor of $\delta$.

$$
\hat{\gamma}_{n, \text { EWMA }}=\delta \gamma_{n-1}+(1-\delta) \hat{\gamma}_{n-1}
$$

EWMA reduces the number of previous measurements to one and has less computational complexity. 
Each of the moving average methods reduces the effect of the measurement errors but introduces more severe staleness effect than the Follower method.

\subsubsection{Linear prediction}

By assuming that the channel quality indicator has a constant first-order derivative across three adjacent transmissions [23], we can predict the ongoing channel quality from the last two channel measurements.

$$
\hat{\gamma}_{n, \text { linear }}=\gamma_{n-1}+\Delta \gamma\left(t_{n}-t_{n-1}\right), \quad \Delta \gamma=\frac{\gamma_{n-1}-\gamma_{n-2}}{t_{n-1}-t_{n-2}}
$$

Here, $\gamma_{n-1}$ and $\gamma_{n-2}$ are the channel measurements at time $t_{n-1}$ and $t_{n-2}$, respectively. This method is more robust to the prediction staleness. However, the errors of the past measurements may make $\Delta \gamma$ twice as noisy, leading to a prediction with an intolerable noise level in some cases.

\subsection{Coherence-aware MMSE first-order prediction}

Our previous discussion reveals that some of the methods are more robust to the measurement errors, while others are more robust to the prediction staleness. For a good prediction, both the measurement errors and staleness should be considered. In this section, we propose a coherence-aware MMSE first-order prediction.

In Section 2.1, we introduced the Rayleigh fading model and its auto-correlation. When conducting the prediction, we need the previous measurements within a time window $T$. From Fig. 1, we see differing dependence between measurements with the same maximum Doppler shift and differing time delay, or with the same time delay and differing maximum Doppler shift. Thus, when selecting the time window $T$, we should take the Doppler shift into account. We can denote $T$ as $T=\frac{\beta}{f_{d}}$, where $f_{d}$ is the Doppler shift that can be estimated by the method we proposed in Section 2. $\beta$ is a constant factor. In our simulation and experiments, we select $\beta=0.064$, which empirically achieves the highest prediction fidelity.

Assuming that within the time window $T$, there are $w$ channel measurements $\gamma_{n-1}, \gamma_{n-2}, \cdots, \gamma_{n-w}$ at time $t_{n-1}, t_{n-2}, \cdots, t_{n-w}$, respectively. From all the measurements within the window, we perform a linear regression first-order curve fit with the constraint of minimum square errors. To do so, we first assume the objective first order curve is $f(t)=a t+b$, where $a$ and $b$ are parameters to be calculated. Then, we have

$$
\gamma^{\prime}{ }_{n-i}=f\left(t_{n-i}\right)=a t_{n-i}+b \quad i=1,2, \cdots, w
$$

The detailed process of computing $a$ and $b$ in (10) can be found in the Appendix 2.

We know that the fading channel does not strictly maintain a constant first-order derivative, especially for long intervals between packets. In a more extreme case, if the interval between the last packet and the ongoing packet exceeds a certain value, the prediction may be uncorrelated with the real channel quality. Considering this interval, we use a weighting factor $\delta$ to weight the preprediction and the channel quality with the maximum probability. Consequently, the estimated channel quality $\hat{\gamma_{n}}$ is:

$$
\hat{\gamma}_{n}=\delta\left(t_{n}-t_{n-1}\right) \cdot \hat{\gamma_{n}}+\left(1-\delta\left(t_{n}-t_{n-1}\right)\right) \cdot \bar{\gamma}
$$

where

$$
\delta(t)= \begin{cases}1-t \cdot f_{d} & \text { if } t<\frac{1}{f_{d}} \\ 0 & \text { otherwise }\end{cases}
$$

In (12), $f_{d}$ is the maximum Doppler shift, and $\bar{\gamma}$ is the channel quality that has the greatest statistical probability of occurrence. For computational simplicity, we approximate $\bar{\gamma}$ as the mean value of the channel quality measurements during the last $10 \mathrm{~s}$.

Note that within the time window $T$, there is the probability of $w \leq 2$. When $w=2$, our algorithm turns out to be the Linear prediction. Similarly, if $w=1$, our algorithm matches the Follower mechanism. For the case of $w=0$, we choose the maximum probability channel quality $\bar{\gamma}$ as our prediction.

In Fig. 9, we show the result of the MMSE first-order channel quality prediction. There is a $-15 \mathrm{~dB}$ measurement error compared to the channel quality. The reconstructed channel response approaches the theoretical curve well. The square errors of the prediction is about $-38 \mathrm{~dB}$ compared to the theoretical one, which means a $-23 \mathrm{~dB}$ accuracy gain.

In order to evaluate the prediction performance of our proposed algorithm under various time intervals between packets, we control the delay between the data packets decoded for channel estimation and the channel feedback packets received by the transmitter. We use a Rayleigh fading channel model to compare our proposed algorithm with three other mechanisms (Follower, EWMA, and Linear prediction), as shown in Fig. 10. We set a Doppler shift of $10 \mathrm{~Hz}$ and set the channel measurement error to $-20 \mathrm{~dB}$ compared to the average channel quality. We can see that the prediction performances based on the time interval seem to follow the same pattern for all prediction algorithms: the prediction error increases as the time interval increases, as shown in Fig. 10a. Our evaluation shows that CIPRA presents the least prediction error of all prediction algorithms. Although similar patterns can be found at both over-selection probability, under-selection probability, and wrong selection probability, as shown in Fig. 10b, c, and d, it is interesting to note that the performance of CIPRA becomes identical to that of Linear prediction at a time interval above $3.6 \mathrm{~ms}$. Considering that the MAC layer design in current and 


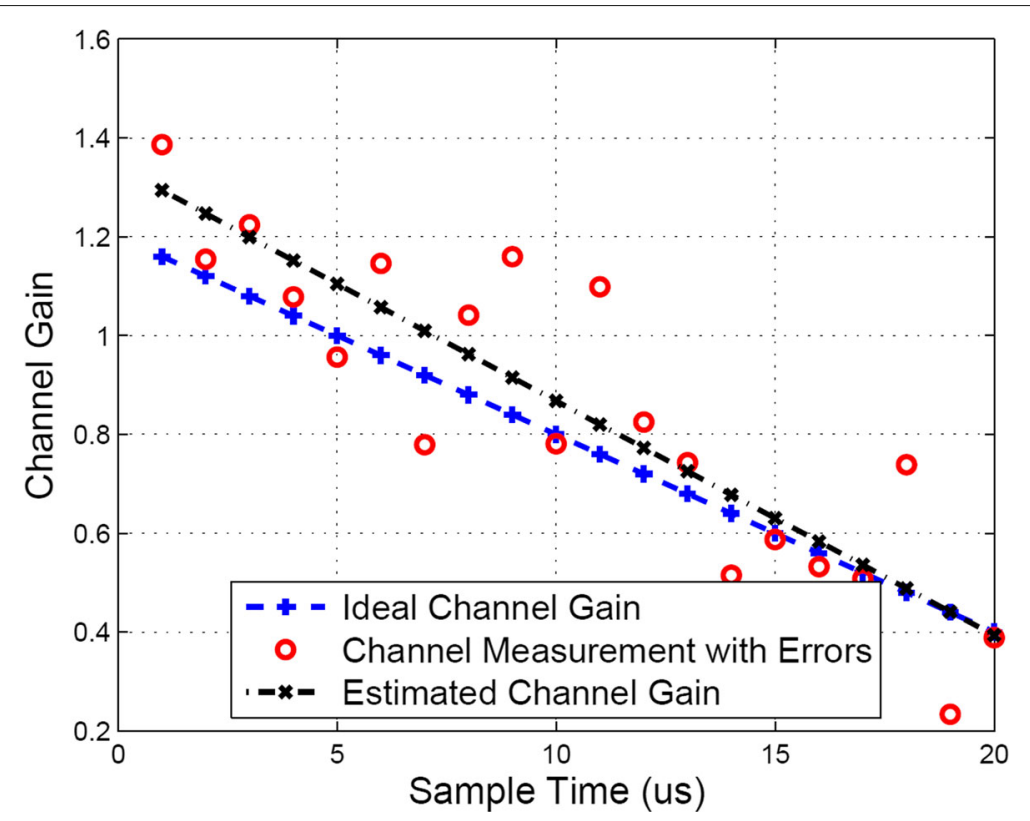

Fig. 9 Channel quality reconstruction using MMSE first-order prediction

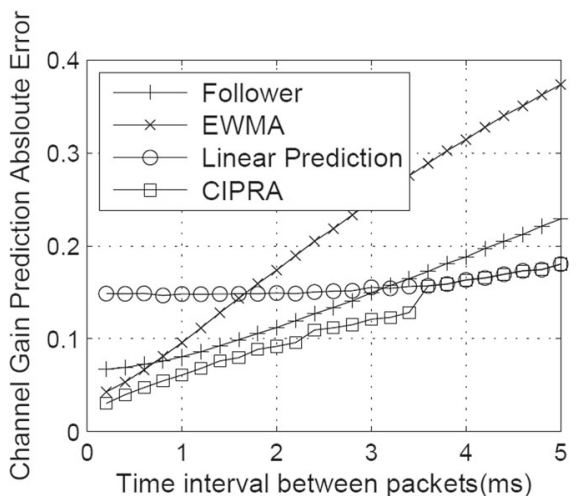

(a)

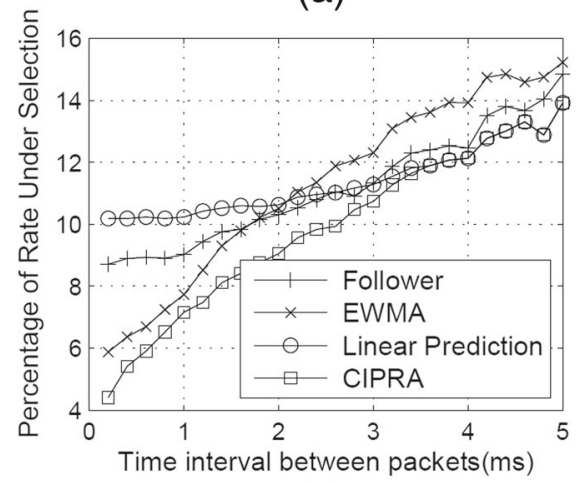

(c)

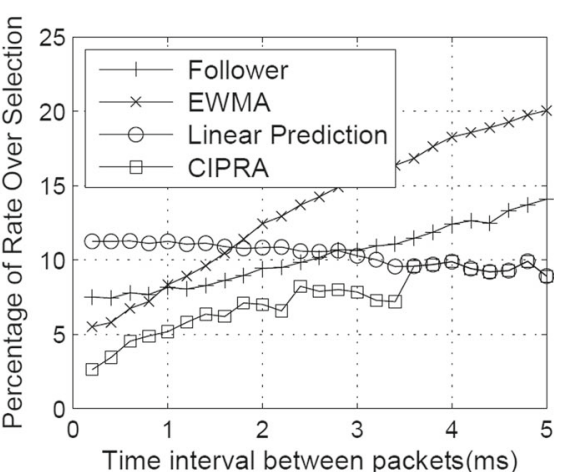

(b)

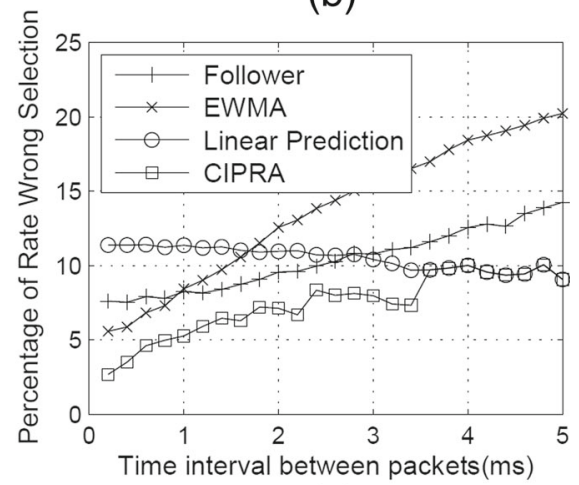

(d)

Fig. 10 a-d Channel quality prediction performances comparison 


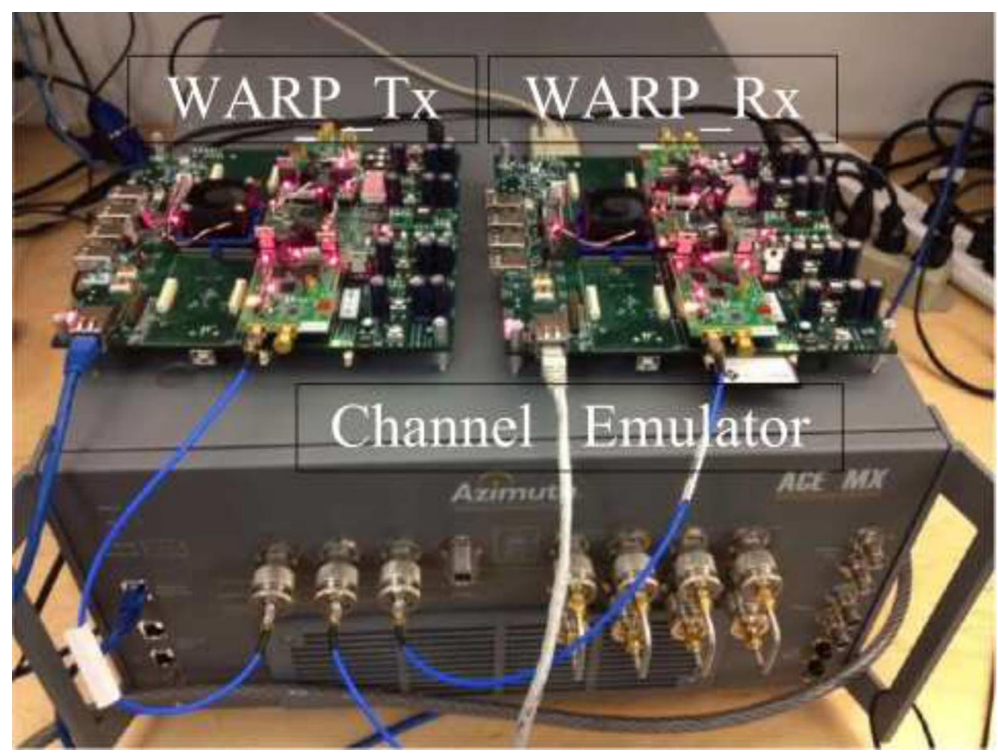

Fig. 11 Channel emulator based evaluation system

future IEEE 802.11 standards requires only smaller time interval between packets, we predict that the impact of CIPRA will only increase with the development of higher frequency band protocols such as those with millimeter wavelengths and smaller timing parameters. For example, a minimum interval between transmission packets, called Short Inter Frame Space (SIFS), has shortened from $28 \mu \mathrm{s}$ in IEEE 802.11-1997 to $16 \mu \mathrm{s}$ in IEEE 802.11n to $3 \mu \mathrm{s}$ in WiGig/IEEE 802.11ad. Moreover, many timing parameters of the backoff process for the carrier sense and channel access are also reduced.

Table 2 Throughput with different combinations of channel indicator and prediction methods

\begin{tabular}{llllll}
\hline \multirow{2}{*}{$\begin{array}{l}\text { Doppler } \\
\text { shift }\end{array}$} & Channel & \multicolumn{4}{l}{ Throughput (Mbps) } \\
\cline { 3 - 6 } $1 \mathrm{~Hz}$ & indicator & Follower & EWMA & Linear & CIPRA \\
\hline \multirow{2}{*}{$2 \mathrm{~Hz}$} & RSSI & 12.59 & 13.41 & 10.88 & 13.88 \\
& SNR & 13.34 & 13.91 & 11.83 & 14.41 \\
& Eff. SNR & 14.19 & 14.55 & 14.05 & 15.06 \\
& RSSI & 11.87 & 12.53 & 10.84 & 13.65 \\
& SNR & 12.98 & 13.28 & 11.89 & 14.26 \\
$5 \mathrm{~Hz}$ & Eff. SNR & 14.16 & 14.41 & 14.18 & 14.95 \\
& RSSI & 11.05 & 11.47 & 10.62 & 13.12 \\
& SNR & 11.91 & 12.55 & 11.85 & 13.80 \\
$10 \mathrm{~Hz}$ & Eff. SNR & 13.76 & 13.85 & 13.68 & 14.52 \\
& RSSI & 10.83 & 11.19 & 10.10 & 12.75 \\
& SNR & 11.87 & 12.46 & 11.20 & 13.31 \\
& Eff. SNR & 13.56 & 13.66 & 13.75 & 14.39 \\
\hline
\end{tabular}

The computational complexity of the proposed algorithm is higher than the other algorithms discussed above. However, it takes less than $1 \mu$ s on the PowerPC embedded on WARP (we use an $80-\mathrm{MHz}$ clock frequency for the PowerPC), which is much less than the DIFS/SIFS time of the transmission. As a result, it does not affect the system throughput.

\section{Hardware setup}

In this section, we describe the implementation of our CIPRA algorithm on the WARP board, a fullycustomized, cross-layer software-defined radio (SDR) platform. Moreover, we use the Azimuth ACE-MX channel emulator to generate controllable channel effects, which allows repeatability of wireless channels over which to test diverse protocols.

\subsection{WARP}

The experiments are carried out using the WARP board, a useful wireless communication system supporting a fully-customized, cross-layer design [11]. We conduct our experimental evaluation based on a full OFDM physical layer design per the IEEE 802.11 PHY frame structure. The design operates in real-time, transmitting and receiving wide-band signals. We implement complete real-time signal processing, synchronization, and control systems in the fabric of the FPGA on WARP. ${ }^{1}$

\footnotetext{
${ }^{1}$ While existing OFDM-based models have existed on the WARP repository (http://warp.rice.edu), they use System Generator to create the design, whereas our design is completely based upon Verilog HDL for system control and efficiency of compilation and real-time operation.
} 


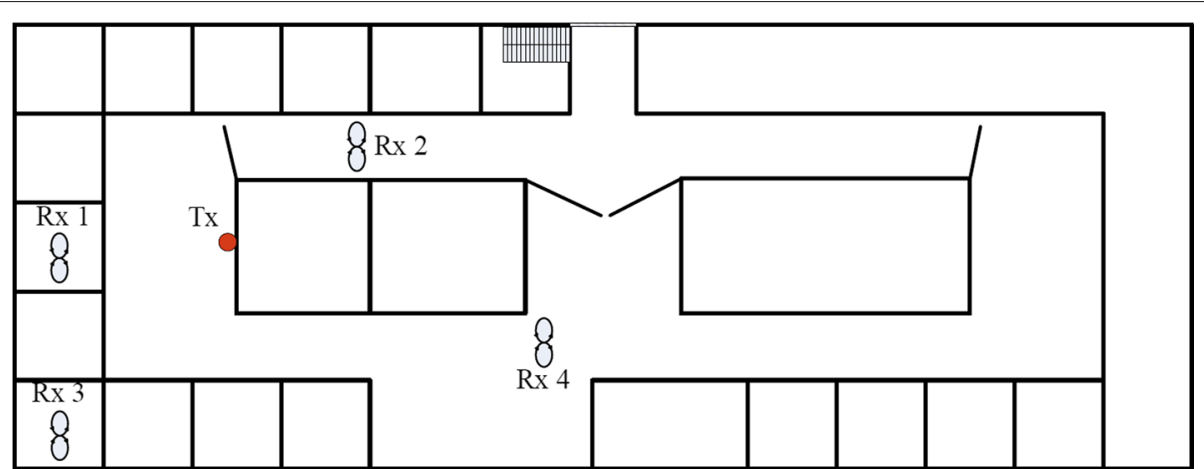

Fig. 12 The eighth floor layout of SMU Expressway Tower

\subsection{Channel emulator}

In addition to the practical in-field wireless channels, we use the Azimuth ACE-MX channel emulator to generate repeatable and controllable channel effects, which isolates the impact of interference and approximates well complex over-the-air channels. Figure 11 illustrates our experimental setup. We use one WARP as the transmitter and another WARP as the receiver. We connect the transmitter and the receiver with RF cabling to the channel emulator. The transmitter sends data packets periodically to the receiver. The receiver measures the channel and feeds the channel indicator back to the transmitter with ACK packets following controllable time interval.

In our evaluation with the channel emulator, we set the packet size to 1536 bytes. We use a two-tap Rayleigh fading channel with an average SNR of $15 \mathrm{~dB}$. Both taps have a $0-\mathrm{dB}$ relative attenuation, and the time delay between the taps is $0.5 \mu \mathrm{s}$. The resulting throughput is shown in Table 2 and indicates that Effective SNR performs best among all three channel indicators. The EWMA method performs better with less of a Doppler shift because there is less staleness when the Doppler shift is low. With an increasing Doppler shift, the linear method becomes comparatively better because of the increasing staleness of Follower and EWMA. SNR-based rate adaptation mechanisms on off-the-shelf devices use the RSSI metric and Follower prediction. Thus, with a Doppler shift of $10 \mathrm{~Hz}$, CIPRA achieves a throughput improvement of $18 \%$ over the off-the-shelf configuration. If further combined with the advanced effective SNR, a total improvement of 33\% could be achieved over the off-the-shelf method. Later, we show that in-field experimental results exceed these gains as the channels become more complex.

\section{Experimental evaluation and discussion}

In this section, we implement the existing channel prediction algorithm and the proposed algorithm on WARP and experimentally compare them in terms of system

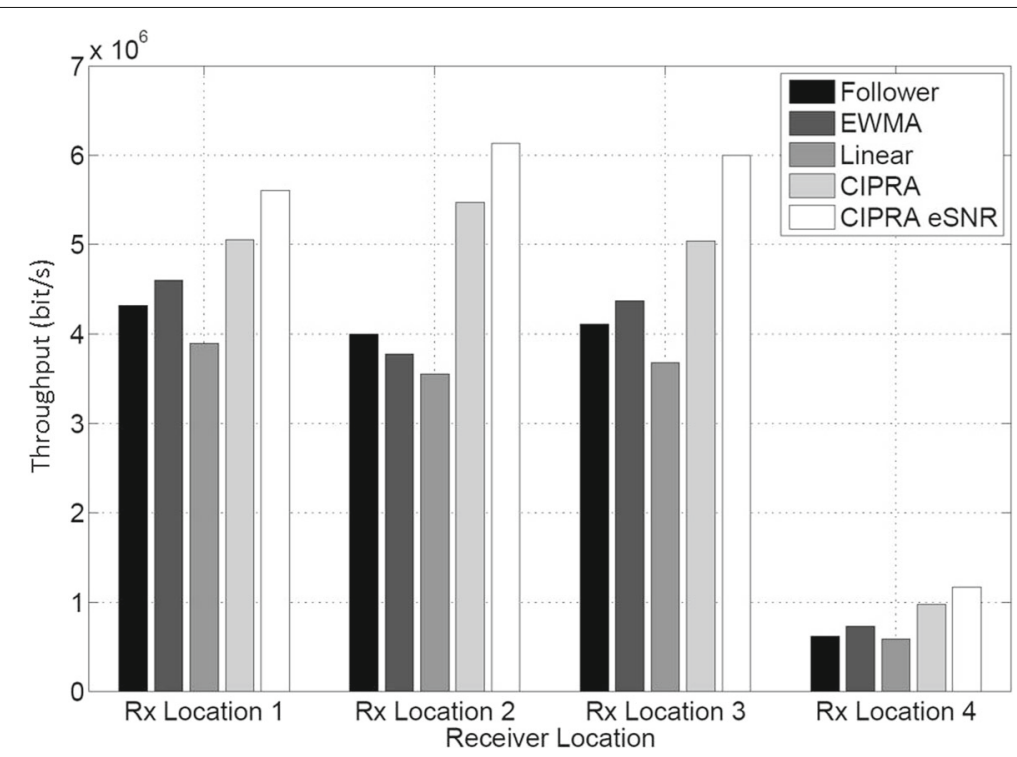

Fig. 13 The indoor experiment result 


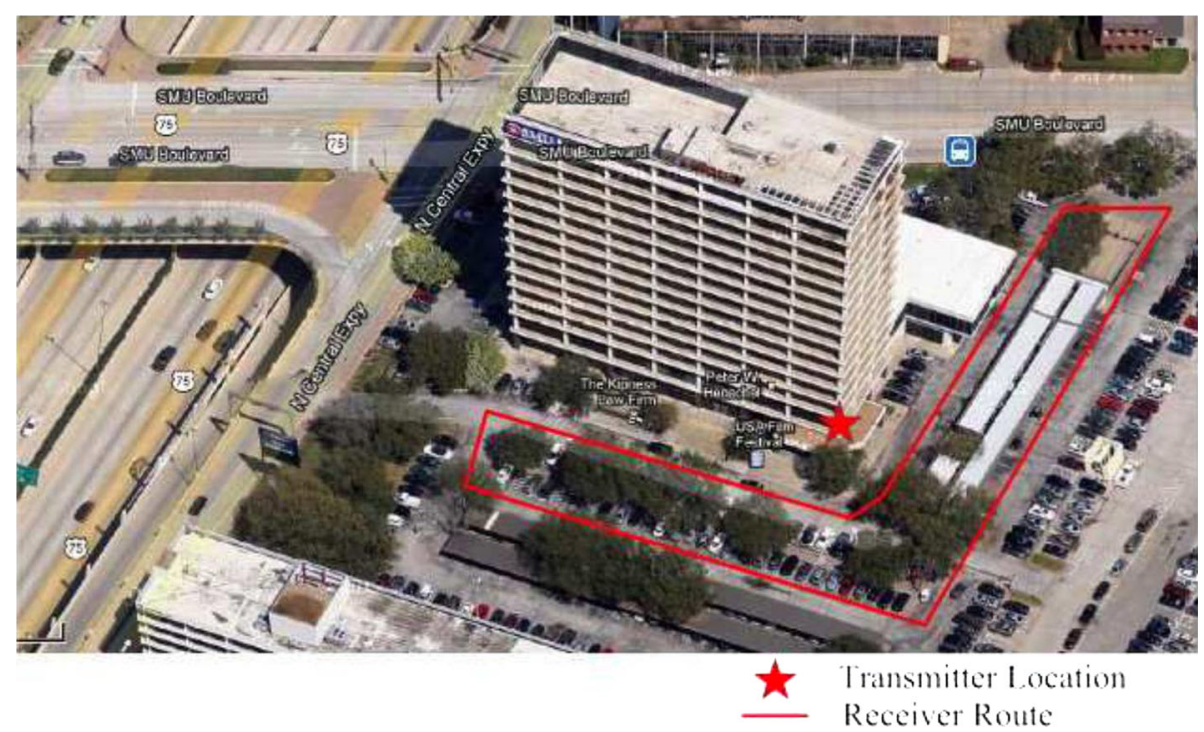

Fig. 14 The experimental environment outside of SMU Expressway Tower

throughput through both indoor pedestrian experiments and outdoor vehicular experiments.

\subsection{Indoor pedestrian test}

We conduct our indoor experiment on the eighth floor of the SMU Expressway Tower (floor plan shown in Fig. 12). Before evaluating the performance of our proposed algorithm with other mechanisms, we take actions to reduce the ambient interference power in our selected measurement locations by disabling or shielding interfering WiFi access points. Yet, the interference cannot be fully eliminated due to the existence of institutional WiFi access points that are beyond our control. We set up two transmitter/receiver pairs, which are operating independently, but simultaneously. Two transceivers use orthogonal channels: $2484 \mathrm{MHz}$ (Ch. 14) and $2462 \mathrm{MHz}$ (Ch. 11), respectively. For each experiment, we run CIPRA on a tx/rx pair, and one of the other three methods on the other $\mathrm{tx} / \mathrm{rx}$ pair. We use RSSI with all four prediction methods to measure the throughput. We also provide additional experiments combining CIPRA and effective SNR to obtain the throughput. We co-locate the transmit antennas and co-locate the receive antennas to ensure the two links have very similar channels. For each comparison, we flip the links back and forth for each experimental trial to remove any unfair advantage between the two channels. The transmitters are located on the table in the lab, and we select four office rooms to put the mobile receivers, as shown in Fig. 12. We randomly move the receiver nodes in each office to create time-varying channels. We show the average throughput of the four methods on the four different locations in Fig. 13. CIPRA greatly outperforms other methods at all four locations with up to a $66 \%$ throughput improvement, with an improved prediction algorithm from the currently one used in practice. When further combined with the advanced Effective SNR, a maximum improvement of $98 \%$ could be achieved over the off-the-shelf method, nearly doubling the achieved throughput. In addition, compared with the experimental results using channel emulator, our evaluation demonstrates the performance improvement of CIPRA in real environments even with the impact of interference.

\subsection{Outdoor vehicular test}

In addition to lab experiments, we also conduct in-field experiments to show the throughput improvement provided by our algorithm for in situ highly mobile scenarios. We perform outdoor experiments in the parking lot of the SMU Expressway Tower (shown in Fig. 14). The transmitter and receiver settings are the same as with the indoor experiments. We place the transmitters in the entrance of the tower and the receivers in a car with the antennas mounted on the roof. We drive the car along the path shown in Fig. 14, with an average speed of $32 \mathrm{~km} / \mathrm{h}$. We also switch the channels of the two tx/rx pairs to remove the unfairness of different channels for each comparison. We show the average throughput of the four methods in Table 3. Due to a higher Doppler shift in the vehicular environment, the Linear method has improved performance. CIPRA also outperforms the three other methods with up to $50 \%$ of the throughput improvement. When

Table 3 Throughput result for outdoor experiment

\begin{tabular}{lllll}
\hline Follower & EWMA & Linear & CIPRA & CIPRA \& eSNR \\
\hline 3.07 Mbps & $3.52 \mathrm{Mbps}$ & $3.95 \mathrm{Mbps}$ & $4.83 \mathrm{Mbps}$ & $5.12 \mathrm{Mbps}$ \\
\hline
\end{tabular}




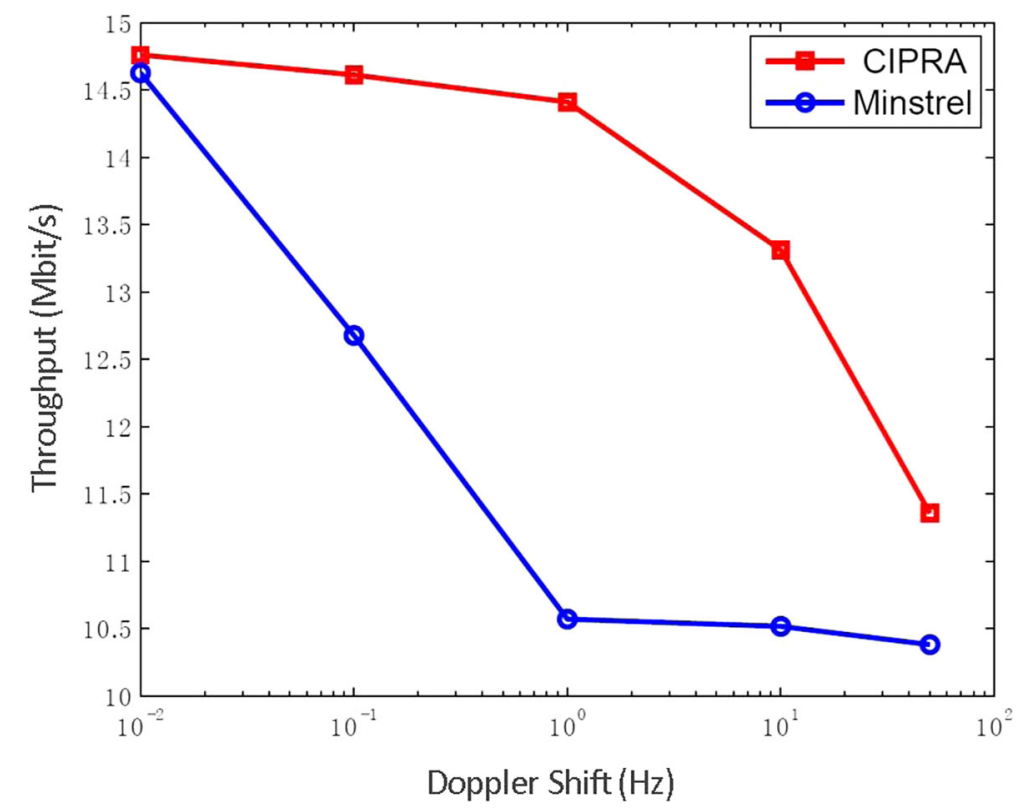

Fig. 15 Throughput comparison between CIPRA and Minstrel

further combined with Effective SNR, a total improvement of $67 \%$ could be achieved over the off-the-shelf method.

5.3 Throughput comparison between CIPRA and Minstrel Minstrel is reported to be one of the best rate adaptation methods based on packet loss/success [37]. We implemented Minstrel according to the specifications in [37] and evaluated the throughput of Minstrel and CIPRA with the same experimental settings as in Section 4.2. We use SNR as the channel indicator for CIPRA and train the CIPRA rate adaptation threshold with a Doppler shift of $10 \mathrm{~Hz}$, corresponding to a walking velocity for $\mathrm{WiFi}$ $2.4 \mathrm{GHz}$ band. The Doppler shift can be approximately by

$$
f_{d}=\frac{v f_{c}}{c}
$$

Here, $v$ is velocity and $c$ is the light speed. Then, we apply the same SNR threshold in all the different Doppler shift cases for CIPRA. We show the throughput of CIPRA and Minstrel in Fig. 15. We can see that, with a very low Doppler shift, CIPRA and Minstrel have similar throughput. However, as the Doppler shift increases, Minstrel degrades faster than CIPRA. This is explained by the long statistical time that composes rate decisions in Minstrel that prevent it from adapting as quickly to fast-fading channels.

\section{Conclusion}

In this paper, we proposed a coherence-aware MMSE first-order prediction algorithm (CIPRA), which considered both the measurement inaccuracy and staleness. Prediction intervals and channel coherence time were jointly considered to select the optimal prediction window. We also implemented a Doppler shift estimation method to assist our prediction algorithm. We compared CIPRA to the traditional channel quality prediction for rate adaptation protocols, performing experiments on an FPGA-based platform over emulated and in-field wireless channels. We showed that our proposed algorithm can provide better prediction fidelity and results in nearly double the throughput versus the current configuration in off-the-shelf devices in the field. Lastly, we estimated that the benefits brought by CIPRA will only increase with the development of next-generation protocols such as those with higher frequencies and higher packet rate.

\section{Appendix 1}

This appendix presents the SNR computation for IEEE 802.11 OFDM systems. As shown in Fig. 6, the long preamble is composed of two identical OFDM symbols with a $1 / 2$ symbol prefix. Denote the OFDM symbol in the long preamble as $X(k), k=0,1, \ldots, K-1$, where $k$ is the sample index in the time domain of one symbol. 
According to the frame structure specified in [35], the long preamble $S(n)$ can be expressed as

$$
S(n)=X\left(\left(n+\frac{K}{2}\right) \bmod (K)\right), \quad n=0,1, \ldots, \frac{5}{2} K-1
$$

where $n$ is the sample index in the long preamble.

We assume this signal passes through a multi-path channel with a time spread of $L, 0 \leq L \leq \frac{K}{4}$. Moreover, we make the assumption that the channel remains constant during one frame slot. Then, the received signal $Y(n)$ with additive white Gaussian noise is

$$
\begin{aligned}
Y(n) & =\sum_{l=0}^{L-1} S(n-l) h(l)+Z(n) \\
& =\sum_{l=0}^{L-1} X\left(\left(n-l+\frac{K}{2}\right) \bmod (K)\right) h(l)+Z(n)
\end{aligned}
$$

where $n=0,1, \ldots, \frac{5}{2} K+l-2$. It is then straightforward that

$$
\begin{aligned}
Y(n+K)= & \sum_{l=0}^{L-1} X\left(\left(n-l+\frac{3 K}{2}\right) \bmod (K)\right) h(l) \\
& +Z(n+K) \\
= & \sum_{l=0}^{L-1} X\left(\left(n-l+\frac{K}{2}\right) \bmod (K)\right) h(l) \\
& +Z(n+K) \\
= & Y(n)+Z(n+K)-Z(n)
\end{aligned}
$$

We then compute the cross-correlation between the last symbol and the first symbol as

$$
\begin{aligned}
P_{S} & =\frac{1}{K} \sum_{n=\frac{K}{2}}^{\frac{3}{2} K-1} Y^{*}(n) Y(n+K) \\
& =\frac{1}{K} \sum_{n=\frac{K}{2}}^{\frac{3}{2} K-1}\left(|Y(n)|^{2}+Y^{*}(n)(Z(n+K)-Z(n))\right)
\end{aligned}
$$

Because $Y(n)$ and $Z(n)$ are uncorrelated, we have

$$
\begin{aligned}
E\left(P_{s}\right)=E\left[\frac{\sum_{n=\frac{K}{2}}^{\frac{3}{2} K-1}\left(Y^{*}(n)(Z(n+K)-Z(n))\right.}{K}\right. \\
\\
\left.+\frac{\left.+\left.Y(n)\right|^{2}\right)}{K}\right] \\
=\frac{1}{K} \sum_{n=\frac{K}{2}}^{\frac{3}{2} K-1} E\left(|Y(n)|^{2}\right)
\end{aligned}
$$

We then compute the auto-correlation on the first symbol as

$$
\begin{aligned}
P_{t} & =\frac{1}{K} \sum_{n=\frac{K}{2}}^{\frac{3}{2} K-1} Y^{*}(n) Y(n) \\
& =\frac{1}{K} \sum_{n=\frac{K}{2}}^{\frac{3}{2} K-1}\left(|Y(n)|^{2}+2 Y^{*}(n) Z(n)+|Z(n)|^{2}\right)
\end{aligned}
$$

Since $Y(n)$ and $Z(n)$ are uncorrelated, we have

$$
\begin{aligned}
E\left(P_{t}\right)= & E\left[\frac { 1 } { K } \sum _ { n = \frac { K } { 2 } } ^ { \frac { 3 } { 2 } K - 1 } \left(|Y(n)|^{2}+2 Y^{*}(n) Z(n)\right.\right. \\
& \left.\left.+|Z(n)|^{2}\right)\right] \\
= & \frac{1}{K} \sum_{n=\frac{K}{2}}^{\frac{3}{2} K-1} E\left(|Y(n)|^{2}+|Z(n)|^{2}\right)
\end{aligned}
$$

Then, $P_{s}$ is the estimated signal power, and $P_{t}$ is the estimated total power. The noise variance will be $P_{t}-P_{s}$. Also, we can calculate the $\mathrm{SNR}$ as $\mathrm{SNR}=\frac{P_{s}}{P_{t}-P_{s}}$.

\section{Appendix 2}

We provide the parameter computation for the first-order SNR prediction. The sum of the square errors between the samples on the curve $\gamma^{\prime} n-i$ and the actual measurements $\gamma_{n-i}$ are

$$
E=\sum_{i=1}^{w}\left(\gamma^{\prime} n-i-\gamma_{n-i}\right)^{2}
$$


Our objective is to find the value of $a$ and $b$ when $E$ achieves its minimum value. We can expand (21) as

$$
\begin{aligned}
E= & \sum_{i=1}^{w}\left(a^{2} t_{n-i}^{2}+2 a\left(b-\gamma_{n-i}\right) t_{n-i}+\left(b-\gamma_{n-i}\right)^{2}\right) \\
= & a^{2} \sum_{i=1}^{w} t_{n-i}^{2}+2 a b \sum_{i=1}^{w} t_{n-i}-2 a \sum_{i=1}^{w} \gamma_{n-i} t_{n-i} \\
& -2 b \sum_{i=1}^{w} \gamma_{n-i}+\sum_{i=1}^{w} \gamma_{n-i}^{2}+\sum_{i=1}^{w} b^{2}
\end{aligned}
$$

Let us use the following notation for ease of expression: $\alpha_{1}=\sum_{i=1}^{w} t_{n-i}^{2}, \alpha_{2}=\sum_{i=1}^{w} t_{n-i}, \alpha_{3}=$ $\sum_{i=1}^{w} \gamma_{n-i} t_{n-i}, \alpha_{4}=\sum_{i=1}^{w} \gamma_{n-i}, \alpha_{5}=\gamma_{n-i}^{2}, \alpha_{6}=w$. Then, we can simply express (22) as

$$
E=\alpha_{1} a^{2}+2 \alpha_{2} a b-2 \alpha_{3} a-2 \alpha_{4} b+\alpha_{5}+\alpha_{6} b^{2}
$$

To find the minimum value of $E$, we take its derivative in terms of $a$ and $b$, respectively. Then, we force both the derivatives to 0 to obtain the following pair of equations:

$$
\left\{\begin{array}{l}
\alpha_{1} a+\alpha_{2} b-\alpha_{3}=0 \\
\alpha_{2} a+\alpha_{6} b-\alpha_{4}=0
\end{array}\right.
$$

From (22), we know that $E \geq 0$ for all $a$ and $b$, which means that there exists a minimum value of $E$. From (22), we can see that $E$ is a convex function of $a$ or $b$. As a result, the solution of $a$ and $b$ in the above equation pairs will enable $E$ to achieve its minimum value. With $a$ and $b$ obtained, we can obtain our pre-estimate of $\hat{\gamma}_{n^{\prime}}$ as

$$
\hat{\gamma}_{n^{\prime}}=f\left(t_{n}\right)=a t_{n}+b
$$

\section{Abbreviations}

ACD: Analog to digital converter; AWGN: Additive white Gaussian noise; BER: Bit error rate; CIPRA: Coherence-aware channel indication and prediction algorithm for rate adaptation; EWMA: Exponential weighted moving average; FFT: Fast Fourier transform; FPGA: Field programmable gate array; ICl: Intercarrier interference; IFFT: Inverse fast Fourier transform; LCR: Level-crossing rate; LOS: Line of sight; LWMA: Linear weighted moving average; MCS: Modulation coding scheme; MMSE: Minimum mean square error; NLOS: None line of sight; OFDM: Orthogonal frequency-division; PHY: Physical layer; RSS: Received signal strength; RSSI: Received signal strength indicator; SDR: Software-defined radio; SIFT: Short Inter Frame Space; SNR: Signal-to-noise ratio; WARP: Wireless open-access research platform; WiFi: Wireless fidelity

\section{Authors' contributions}

YD and PH conceived the framework design and performed the data analysis. YS improved the work and took charge of all the work of paper submission. DR and $\mathrm{JC}$ reviewed and revised the manuscript. All authors have read and approved the final manuscript.

\section{Funding}

This work was in part supported by NSF grants: CNS-1150215, CNS-1823304, and CNS-1526269.

\section{Competing interests}

The authors declare that they have no competing interests.

\section{Author details}

${ }^{1}$ Broadcom Limited, Irvine, CA, USA. ${ }^{2}$ Samsung Research America, Plano, TX, USA. ${ }^{3}$ Department of Electrical Engineering, Southern Methodist University, Dallas, TX, USA.

Received: 23 August 2018 Accepted: 18 July 2019

Published online: 07 August 2019

\section{References}

1. Y. Shi, R. Enami, J. Wensowitch, J. Camp, Uabeam: Uav-based beamforming system analysis with in-field air-to-ground channels, 201815 th Annual IEEE International Conference on Sensing, Communication, and Networking (SECON), (2018)

2. X. Liu, M. Jia, Z. Na, W. Lu, F. Li, Multi-modal cooperative spectrum sensing based on dempster-shafer fusion in 5 g-based cognitive radio, IEEE ACCESS, (2018)

3. X. Liu, X. Zhang, M. Jia, L. Fan, W. Lu, X. Zhai, 5g-based green broadband communication system design with simultaneous wireless information and power transfer, IEEE ACCESS, (2018)

4. X. Liu, M. Jia, X. Zhang, W. Lu, A novel multi-channel internet of things based on dynamic spectrum sharing in $5 \mathrm{~g}$ communication, IEEE Internet of Things Journal, (2018)

5. J. C. Bicket, Bit-rate selection in wireless networks, Massachusetts Institute of Technology Library, (2005)

6. A. Ilori, Z. Tang, J. He, Y. Li, Throughput-based rate adaptation algorithm for IEEE 802.11 vehicle networks. Netw. IET. 4(2), 111-118 (2015)

7. C.-Y. Li, C. Peng, P. Cheng, S. Lu, X. Wang, F. Ren, T. Wang, An energy efficiency perspective on rate adaptation for $802.11 \mathrm{n}$ nic. Mob. Comput. IEEE Trans. PP(99), 1-1 (2015)

8. Z. Dong, P. Fan, E. Panayirci, X. Lei, Conditional power and rate adaptation for MQAM/OFDM systems under CFO with perfect and imperfect channel estimation errors. Veh. Technol. IEEE Trans. 64(11), 5042-5055 (2015)

9. G. Wang, S. Zhang, K. Wu, Q. Zhang, L. M. Ni, TiM: Fine-grained rate adaptation in WLANs. Mob. Comput. IEEE Trans. 15(3), 748-761 (2016)

10. C.-Y. Li, C. Peng, S. Lu, X. Wang, R. Chandra, Latency-aware rate adaptation in $802.11 \mathrm{n}$ home networks, IEEE INFOCOM, (2015)

11. J. Camp, E. Knightly, Modulation rate adaptation in urban and vehicular environments: Cross-layer implementation and experimental evaluation. Netw. IEEE/ACM Trans. 18(6), 1949-1962 (2010)

12. Y. Du, P. Huang, D. Rajan, J. Camp, Cipra: Coherence-aware channel indication and prediction for rate adaptation, International Wireless Communications and Mobile Computing Conference (IWCMC), (2013)

13. M. Vutukuru, H. Balakrishnan, K. Jamieson, Cross-layer wireless bit rate adaptation, ACM SIGCOMM, (2009)

14. D. Halperin, W. Hu, A. Sheth, D. Wetherall, Predictable 802.11 packet delivery from wireless channel measurements, ACM SIGCOMM, (2010)

15. S. Sen, N. Santhapuri, R. R. Choudhury, S. Nelakuditi, Accurate: Constellation based rate estimation in wireless networks, SENIX NSDI, (2010)

16. G. Judd, X. Wang, P. Steenkiste, Efficient channel-aware rate adaptation in dynamic environments, the 6th international conference on Mobile systems, applications, and services, (2008)

17. Y. Shi, Improving the performance of RSS detection using wireless open-source platforms, Texas Symposium on Wireless and Microwave Circuits and Systems (TWMCS), (2015)

18. P. G. Xi Chen, D. Qiao, Ram: Rate adaptation in mobile environments. Mob. Comput. IEEE Trans. 11(3), 464-477 (2012)

19. S. Abdallah, S. D. Blostein, Rate adaptation using long range channel prediction based on discrete prolate spheroidal sequences, Signal Processing Advances in Wireless Communic ations (SPAWC), (2014)

20. C. Min, N. Chang, J. Cha, J. Kang, MIMO-OFDM downlink channel prediction for IEEE 802.16e systems using Kalman filter, IEEE 2007 Wireless Communications and 848 Networking Conference (WCNC), (2007)

21. K. Farkas, K. Ruf, T. Hossmann, B. Plattner, Pattern matching based link quality prediction in wireless mobile ad hoc networks, ACM International Conference on Modelling, Analysis and Simulation of Wireless and Mobile Systems (MSWiM), (2006)

22. A. Wapf, M. R. Souryal, Measuring indoor mobile wireless link quality, IEEEICC, (2009)

23. P. Casari, B. Tomasi, K. Pelekanakis, M. Chitre, M. Zorzi, Performance evaluation of SNR prediction schemes in acoustic communication systems using variable-rate modulation, IEEE Underwater Acoustic Measurement (UAM), (2011) 
24. W. C. Jakes, Microwave mibile commmunications. (Wiley, New York, 1975)

25. G. L. Stuber, Principles of mobile communications. (MA:Kluwer, Norwell, 2000)

26. W. Zhou, W. H. Lam, A novel method of Doppler shift estimation for OFDM systems, IEEE Military Communications Conference (MILCOM), (2008)

27. T. S. Rappaport, Wireless communications: Principles and practice. (PTR, Prentice Hall, 2001)

28. G. Park, D. Hong, C. Kang, Level crossing rate estimation with Doppler adaptive noise suppression technique in frequency domain, IEEE Vehicular Technology Conference VTC, (2003)

29. S. G. Johnson, M. Frigo, A modified split-radix FFT with fewer arithmetic operations. Signal. Process. IEEE Trans. 55(1), 111-119 (2007)

30. Wild Packets White Paper (2018). http://www.wildpackets.com/elements/ whitepapers/Converting_Signal_Strength.pdf

31. C. Fernandes, J. Mota, Favier, Gerard, Cancellation of nonlinear inter-carrier interference in ofdm systems with nonliear power-amplifiers. (Springer, Berlin/Heidelberg, 2010)

32. M. L. Huang, J. Lee, S. Park, An iterative SNR estimator for link adaptation in IEEE 802.11n System, the 23rd International Technical Conference on Circuits/Systems, Computers and Communications, (2008)

33. T. Yucek, H. Arslan, MMSE noise power and SNR estimation for OFDM systems, Sarnoff Symposium, (2006)

34. Z. Cvetkovic, V. Tarokh, S. Yoon, On frequency offset estimation for OFDM Wirel. Commun. IEEE Trans. 12(3), 1062-1072 (2013)

35. J. Goldberg, IEEE standard for W LAN- part 11: Wireless LAN medium access control (MAC) and physical layer (PHY) specifications, IEEE Standard Committee (2016)

36. A. Goldsmith, Wireless communications, Cambridge University Press, (2005)

37. J. Otaku, Minstrel Rate Adaptation Algorithm Documentation. http://madwifiproject. org/browser/madwifi/trunk/ath_rate/minstrel/minstrel.txt

\section{Publisher's Note}

Springer Nature remains neutral with regard to jurisdictional claims in published maps and institutional affiliations.

\section{Submit your manuscript to a SpringerOpen ${ }^{\circ}$ journal and benefit from:}

- Convenient online submission

- Rigorous peer review

- Open access: articles freely available online

- High visibility within the field

- Retaining the copyright to your article

Submit your next manuscript at $\boldsymbol{s p r i n g e r o p e n . c o m ~}$ 